\title{
DETERMINATION OF MATERIAL PARAMETERS OF ISOTROPIC AND ANISOTROPIC HYPER-ELASTIC MATERIALS USING BOUNDARY MEASURED DATA
}

\author{
Maedeh Hajhashemkhani, Mohammad R. Hematiyan \\ Shiraz University, Department of Mechanical Engineering, Shiraz, Iran \\ e-mail: maedeh.hajhashemkhani@gmail.com; mhemat@shirazu.ac.ir
}

\begin{abstract}
Identification of mechanical properties of isotropic and anisotropic materials that demonstrate non-linear elastic behavior, such as rubbers and soft tissues of human body, is critical for many industrial and medical purposes. In this paper, a method is presented to obtain the mechanical constants of Mooney-Rivlin and Holzapfel hyper-elastic material models which are employed to describe the behavior of isotropic and anisotropic hyper-elastic materials, respectively. By using boundary measured data from a sample with non-standard geometry, and by using an iterative inverse analysis technique, the material constants are obtained. The method uses the results of different experiments simultaneously to obtain the material parameters more accurately. The effectiveness of the proposed method is demonstrated through three examples. In the two first examples, the simulated measured data are used, while in the third example, the experimental data obtained from a polyvinyl alcohol sample are used.
\end{abstract}

Keywords: hyper-elstic material, inverse analysis, anisotropic, polyvinyl alcohol

\section{Introduction}

Many materials such as rubbers, elastomers and tissues of human body experience large deformation under small loads. Further, some materials such as wood, fiber-reinforced composites and some body tissues like arterial walls, show anisotropic behavior in addition to large elastic deformation due to the presence of preferred directions in their structure. The large strains in the response of these materials clarify that their mechanical behavior is nonlinear, and getting back to the reference configuration after load removal demonstrates their elastic response. Because of the wide use of these materials in industry and the crucial role of different tissues in human body, presenting a model with known parameters that can predict the mechanical behavior of these materials is essential. When these materials experience small deformations (less than 2 to 5 percent), their mechanical behavior can be modeled using common linear elastic models (Czabanowski, 2010), but under large deformations, their mechanical response must be represented by nonlinear models such as hyper-elastic material models. Therefore, many efforts have been made to present constitutive laws that model the behavior of these materials properly. Pamidi and Advani (1987) proposed nonlinear constitutive relations for human brain tissue. Fung (1993) showed that the elastic properties of rabbits' mesentery could be simply modeled as an exponential function. Holzapfel and Gasser (2000) considered arterial walls as thin-walled cylinders and presented a constitutive equation for describing their behavior. They obtained the numerical values of the constants of their model using experimental data. A constitutive law for arterial layers with distributed collagen fiber orientation was presented in the work of Gasser et al. (2006).

Many researchers used hyper-elastic models to predict material behavior especially for human body tissues. Moulton et al. (1995) used a combination of finite element model, nonlinear 
optimization algorithm, and a set of strains obtained by magnetic resonance imaging (MRI) to determine passive myocardial material properties. Miller and Chinzei (1997) conducted pressure experiments on brain tissue to model the destructions that may happen during brain surgery. They obtained force-displacement diagrams from the experiments. Then, they computed the unknown constants for different hyper-elastic models using the least squares method. Krouskop et al. (1998) conducted some pressure experiments on different parts of breast and prostate tissue, such as fat, and obtained Young's modules for each part.

Hartman (2001) conducted some tension and torsion tests on different samples made of rubber to identify their properties based on incompressible Rivlin's hyper-elastic model. Ogden et al. (2004) used a non-linear least squares optimization method to obtain the constants of hyper-elastic models for incompressible materials by fitting experimental data to their model. In the work of $\mathrm{Hu}$ and Desai (2004), a tissue indentation test was conducted on liver to identify its biomechanical properties.

Balaraman et al. (2005) conducted different experiments on 19 samples of muscle tissue and used the experimental data in a finite element analysis. They obtained mechanical properties of the muscle by an inverse method using Taguchi's approach. Mehrabian (2008) estimated the constants of an incompressible hyper-elastic material based on three different energy functions. Some samples made of polyvinyl alcohol were used in pressure experiments. The tests were modeled in the finite element analysis and the unknown constants were computed using the least square method.

Ahn et al. (2008) conducted biomechanical experiments on macro and micro liver samples to characterize their mechanical behavior using a nonlinear least squares method and an inverse finite element (FE) method based on a parameter estimation algorithm. In the work of MesaMúnera et al. (2012), several hyper-elastic models were used to predict and model mechanical behavior of a silicone rubber. They conducted a uniaxial compression test on a phantom with mechanical properties close to brain tissue. They could obtain force-displacement diagrams for compression tests on the phantom.

Rauchs et al. (2010) used a depth-sensing spherical indentation technique to determine the parameters of different rubber materials by using an inverse method based on a gradient-based numerical optimization method. Czabanowski (2010) conducted some pressure experiments on machinery elastomers to obtain their material properties. Czabanowski obtained force-displacement diagrams first. Then, by using a function in ABAQUS finite element software, he determined the constants of Mooney-Rivlin model for the materials.

Abyaneh et al. (2013) used an inverse method to obtain viscoelastic material properties of porcine cornea by performing tension and indentation tests. They used a sample with possible maximum length for the tension test. Using the results of the tension test, material properties for an isotropic viscoelastic material were obtained using a curve fitting procedure. Due to insufficiency of this model, to match the indentation test results, an anisotropic model with the results of the indentation test was also used in an inverse algorithm to obtain anisotropic parameters. Parameters of the isotropic model were the initial guesses for the inverse algorithm. Baker and Shrot (2013) proposed a new method for inverse identification of material parameters. They used auxiliary quantities to describe material behavior.

In all of the works reviewed above, the sample that was used to obtain the measurement data was cut to a cubic or cylindrical shape to provide standard test specimens. Therefore, these methods cannot be applicable to find the material constants of a hyper-elastic material with a non-standard shape nondestructively. The inverse method presented in this paper uses the measured displacement data from a sufficient number of sampling points on the original hyperelastic body to obtain unknown material constants. If the properties of the material are dependent on size and geometry, the properties, which are obtained by this method, are suitable for that special shape. Unlike previous researches, the method presented in this research uses the 
results of different experiments simultaneously to obtain the material parameters more accurately. This inverse problem is highly nonlinear and encounters various difficulties. Simultaneous use of measured data from several experiments with different load cases reduces the ill-posedness of the inverse problem. It is observed that using the measured data from several experiments results in a better solution than the case where only the measured data from one experiment is used. The Mooney-Rivlin and Holzapfel models are considered for isotropic and anisotropic materials, respectively. The Tikhonov regularization method is used in the inverse analysis. A method to determine the initial guesses for the unknown constants is also presented. The inverse method needs a sensitivity analysis, which is carried out using the finite element method (FEM).

\section{Materials modeling}

\subsection{Hyper-elastic materials}

For hyper-elastic materials, the stress-strain relations are determined using the strain energy density function $\psi$ which is defined in terms of a deformation gradient or strain tensor. The derivative of $\psi$ with respect to a component of strain gives its corresponding stress component (Holzapfel, 2000)

$$
S_{i j}=\frac{\partial \psi}{\partial \varepsilon_{i j}}
$$

where $\mathbf{S}$ is the second Piola-Kirchhoff stress tensor and $\boldsymbol{\varepsilon}$ is the Lagrangian strain tensor defined as follows

$$
\varepsilon_{i j}=\frac{1}{2}\left(C_{i j}-\delta_{i j}\right)
$$

In Eq. (2.2), $\mathbf{C}$ is the right Cauchy-Green deformation tensor $\left(\mathbf{C}=\mathbf{F}^{\mathrm{T}} \mathbf{F}\right) . \mathbf{F}$ is the deformation gradient, which can be expressed in terms of the displacement vector $\mathbf{u}(\mathbf{F}=\nabla \mathbf{u}+\mathbf{I})$.

Local shape deformation of a material element is described by $\mathbf{F}$. The strain energy function is usually a function of deformation gradient $\mathbf{F}$. Indeed, the function $\psi$ is expressed in terms of the right Cauchy-Green deformation tensor, i.e., $\psi=\psi(\mathbf{C})$. The following relation holds between the second Piola-Kirchhoff and Cauchy stress tensors (Holzapfel, 2000)

$$
\mathbf{S}=J \mathbf{F}^{-1} \boldsymbol{\sigma} \mathbf{F}^{-T}
$$

where $J=\operatorname{det} \mathbf{F}$. Therefore, according to Eqs. (2.1), (2.2) and (2.3) the Cauchy stress tensor is defined as follows

$$
\boldsymbol{\sigma}=2 J^{-1} \mathbf{F} \frac{\partial \psi}{\partial \mathbf{C}} \mathbf{F}^{\mathrm{T}}
$$

For an isotropic material, $\psi$ is dependent on $\mathbf{C}$ based on its invariants. The invariants of $\mathbf{C}$ are

$$
I_{1}=\operatorname{tr} \mathbf{C} \quad I_{2}=\frac{1}{2}\left[(\operatorname{tr} \mathbf{C})^{2}-\operatorname{tr} \mathbf{C}^{2}\right] \quad I_{3}=\operatorname{det} \mathbf{C}
$$

For an isotropic material $\psi$ is just dependent on $I_{1}, I_{2}$ and $I_{3}$ (Holzapfel and Ogden, 2010) and the Cauchy stress equation is expanded as follows

$$
\boldsymbol{\sigma}=2 J^{-1}\left(\mathbf{F} \frac{\partial \psi}{\partial I_{1}} \frac{\partial I_{1}}{\partial \mathbf{C}} \mathbf{F}^{\mathrm{T}}+\mathbf{F} \frac{\partial \psi}{\partial I_{2}} \frac{\partial I_{2}}{\partial \mathbf{C}} \mathbf{F}^{\mathrm{T}}+\mathbf{F} \frac{\partial \psi}{\partial I_{3}} \frac{\partial I_{3}}{\partial \mathbf{C}} \mathbf{F}^{\mathrm{T}}\right)
$$


By obtaining the derivatives of the invariants with respect to $\mathbf{C}$ (Holzapfel, 2000) and knowing that $\mathbf{B}=\mathbf{F F}^{\mathrm{T}}$ Eq. (2.6) yields to

$$
\boldsymbol{\sigma}=2 J^{-1}\left[\psi_{1} \mathbf{B}+\psi_{2}\left(I_{1} \mathbf{B}-\mathbf{B}^{2}\right)+I_{3} \psi_{3} \mathbf{I}\right]
$$

where $\psi_{i}=\partial \psi / \partial I_{i}$. For an incompressible isotropic material $I_{3}=\operatorname{det} \mathbf{F}=1$ and the Cauchy stress tensor is modified as follows (Holzapfel, 2000)

$$
\boldsymbol{\sigma}=-p \mathbf{I}+2 \mathbf{F} \frac{\partial \psi}{\partial \mathbf{C}} \mathbf{F}^{\mathrm{T}}
$$

where $p$ is a scalar identified as hydrostatic pressure. Therefore, the Cauchy stress tensor for an incompressible material with respect to $\mathbf{B}$ is expressed as follows

$$
\boldsymbol{\sigma}=-p \mathbf{I}+2 \psi_{1} \mathbf{B}+2 \psi_{2}\left(I_{1} \mathbf{B}-\mathbf{B}^{2}\right)
$$

\subsection{Isotropic hyper-elastic materials}

The polynomial hyper-elastic model is a proper model for rubbers and other soft materials with isotropic behavior. In this research, the Mooney-Rivlin hyper-elastic model, which is obtained from the polynomial model, is used to predict the mechanical behavior of isotropic materials. In this model, density of strain energy for an incompressible material is a function of the first and second invariants of the left Cauchy-Green deformation tensor. The strain energy function for the polynomial model is defined as follows (Rivlin and Saunder, 1951)

$$
\psi=\sum_{i, j=0}^{n} A_{i j}\left(I_{1}-3\right)^{i}\left(I_{2}-3\right)^{j}
$$

where $A_{i j}$ are the constants of the material and $A_{00}=0$. The Mooney-Rivlin strain energy function is obtained by considering $n=1$ and $A_{11}=0$ in equation (2.10), i.e.

$$
\psi=A_{10}\left(I_{1}-3\right)+A_{01}\left(I_{2}-3\right)
$$

By considering relations (2.9) and (2.11), the Cauchy stress for the Mooney-Rivlin hyper-elastic material is obtained as follows

$$
\boldsymbol{\sigma}=-p \mathbf{I}+2 A_{10} \mathbf{B}+2 A_{01}\left(I_{1} \mathbf{B}-\mathbf{B}^{2}\right)
$$

\subsection{Anisotropic hyper-elastic materials}

In the structure of human soft tissues, the presence of collagen fibers (Unnikrishnan, 2012) causes the material to have one or more preferred direction. This preferred direction is presented here by $\mathbf{M}$. In this case, the strain energy density is a function of both $\mathbf{C}$ and $\mathbf{M}$. Two more dependent pseudo invariants are defined for these materials (Holzapfel and Gasser, 2000)

$$
I_{4}=\mathbf{M}(\mathbf{C M}) \quad I_{5}=\mathbf{M}\left(\mathbf{C}^{2} \mathbf{M}\right)
$$

where, for example, $\mathbf{C M}$ represents the action of the second order tensor $\mathbf{C}$ on the vector $\mathbf{M}$.

For an incompressible material reinforced with one family of fibers, $\psi$ is dependent on $I_{1}$, $I_{2}, I_{4}$ and $I_{5}$. In this case, the Cauchy stress has two additional terms which show the effect of anisotropy. Therefore, the Cauchy stress tensor can be expressed as follows (Holzapfel and Ogden, 2010)

$$
\boldsymbol{\sigma}=-p \mathbf{I}+2 \psi_{1} \mathbf{B}+2 \psi_{2}\left(I_{1} \mathbf{B}-\mathbf{B}\right)^{2}+2 \psi_{4} \mathbf{m} \otimes \mathbf{m}+2 \psi_{5}[\mathbf{m} \otimes \mathbf{B m}+\mathbf{B m} \otimes \mathbf{m}]
$$


where $\otimes$ denotes the dyadic product of two vectors and $\mathbf{m}=\mathbf{F M}$ is the deformed form of the vector $\mathbf{M}$ in current configuration. For some tissues like arterial walls, two families of fibers with different directions can be detected within the tissue. $\mathbf{M}^{\prime}$ is considered to be the unit vector in the direction of the second family of fibers. In this case, three more invariants are considered, which are expressed as follows (Holzapfel and Ogden, 2010)

$$
I_{6}=\mathbf{M}^{\prime}\left(\mathbf{C M}^{\prime}\right) \quad I_{7}=\mathbf{M}^{\prime}\left(\mathbf{C}^{2} \mathbf{M}^{\prime}\right) \quad I_{8}=\left[\mathbf{M}\left(\mathbf{C M}^{\prime}\right)\right]\left(\mathbf{M M}^{\prime}\right)
$$

In this case, the Cauchy stress is expressed as follows (Holzapfel and Ogden, 2010)

$$
\begin{aligned}
\boldsymbol{\sigma}= & -p \mathbf{I}+2 \psi_{1} \mathbf{B}+2 \psi_{2}\left(I_{1} \mathbf{B}-\mathbf{B}\right)^{2}+2 \psi_{4} \mathbf{m} \otimes \mathbf{m}+2 \psi_{5}[\mathbf{m} \otimes \mathbf{B m}+\mathbf{B m} \otimes \mathbf{m}] \\
& +2 \psi_{6} \mathbf{m}^{\prime} \otimes \mathbf{m}^{\prime}+2 \psi_{7}\left[\mathbf{m}^{\prime} \otimes \mathbf{B m}^{\prime}+\mathbf{B} \mathbf{m}^{\prime} \otimes \mathbf{m}^{\prime}\right]+2 \psi_{8}\left(\mathbf{M} \otimes \mathbf{M}^{\prime}\right)\left(\mathbf{m} \otimes \mathbf{m}^{\prime}+\mathbf{m}^{\prime} \otimes \mathbf{m}\right)
\end{aligned}
$$

In this research, the Holzapfel's strain energy function for anisotropic materials is used. This function is described as follows (Holzapfel and Ogden, 2010)

$$
\psi=R_{10}\left(I_{1}-3\right)+\frac{k_{1}}{k_{2}}\left\{\exp \left[k_{2}\left(I_{4}^{*}-1\right)^{2}\right]-1\right\}
$$

$R_{10}, k_{1}, k_{2}$ and $\kappa$ are material constants and $I_{4}^{*}$ is defined as follows

$$
I_{4}^{*}=\kappa I_{1}+(1-3 \kappa) I_{4}
$$

In this model, it is assumed that the direction of each family of fibers is dispersed around a mean direction. This dispersion is shown by $\kappa(0 \leqslant \kappa \leqslant 1 / 3)$ (Holzapfel and Ogden, 2010). By using relations (2.16), (2.17) and (2.18), the Cauchy stress tensor can be obtained.

\section{Computation of unknown material parameters of hyper-elastic materials}

In this paper, an inverse method is used to obtain the constants of hyper-elastic material models. The inverse analysis is used to convert the measured data to some information about the material or system under study. In the inverse analysis, the outputs of the system may be available from an experiment but loading parameters, material properties, geometry of structure, boundary conditions or a combination of these factors have to be determined (Liu and Han, 2003). In the present study, unknowns are constants of hyperelastic materials. These unknowns are found using the measured displacements at some sampling points on the boundary of the body. The measured displacements are collected from a few load cases (for example, 3 cases). Then by using the inverse analysis in an iterative process, the constants are obtained. A proper initial guess is required for the iterative process.

\subsection{Inverse analysis}

In this section, the inverse formulation for evaluation of constants of isotropic and anisotropic hyper-elastic material models is presented.

For an incompressible material, the Mooney-Rivlin hyper-elastic model constants are $A_{10}$ and $A_{01}$. Therefore, the vector of unknowns for the problem is defined as follows

$$
\mathbf{x}=\left[\begin{array}{ll}
x_{1} & x_{2}
\end{array}\right]^{\mathrm{T}}
$$

where $x_{1}=A_{10}$ and $x_{2}=A_{01}$. For the Holzapfel hyper-elastic model, these constants are $R_{10}$, $k_{1}, k_{2}$ and $\kappa$. The vector of unknowns for this case is defined as follows

$$
\mathbf{x}=\left[\begin{array}{llll}
x_{1} & x_{2} & x_{3} & x_{4}
\end{array}\right]^{\mathrm{T}}=\left[\begin{array}{llll}
R_{10} & k_{1} & k_{2} & \kappa
\end{array}\right]^{\mathrm{T}}
$$


In order to obtain material constants, a few experiments (load cases) are performed on the material. These experiments must be different with each other to provide suitable data for the inverse analysis. The number of these experiments is 2,3 or more. In this work, 3 load cases are considered for describing the formulation.

In each experiment, the displacement at some boundary points is measured and restored in vectors $\mathbf{Y}^{(1)}, \mathbf{Y}^{(2)}$ and $\mathbf{Y}^{(3)}$. If they are $N_{1}, N_{2}$ and $N_{3}$ measured data in load cases 1,2 and 3, respectively, $\mathbf{Y}^{(i)}(i=1,2,3)$ are defined as follows

$$
\mathbf{Y}^{(i)}=\left[\begin{array}{llll}
Y_{1}^{(i)} & Y_{2}^{(i)} & \cdots & Y_{N_{i}}^{(i)}
\end{array}\right] \quad i=1,2,3
$$

By solving the problem numerically using the estimated constants, the displacement vector at sampling points is obtained and stored in $\mathbf{D}^{(1)}, \mathbf{D}^{(2)}$ and $\mathbf{D}^{(3)}$ which are defined as follows

$$
\mathbf{D}^{(i)}=\left[\begin{array}{llll}
D_{1}^{(i)} & D_{2}^{(i)} & \cdots & D_{N_{i}}^{(i)}
\end{array}\right] \quad i=1,2,3
$$

In order to find the constants of the material, the Tikhonov regularization method is used. In this method, the objective function $\Pi$ is defined as follows

$$
\Pi=(\mathbf{Y}-\mathbf{D})^{\mathrm{T}}(\mathbf{Y}-\mathbf{D})+\alpha \mathbf{X}^{\mathrm{T}} \mathbf{X}
$$

where the vectors $\mathbf{Y}$ and $\mathbf{D}$ are defined as follows

$$
\mathbf{Y}=\left[\begin{array}{lll}
\mathbf{Y}^{(1)} & \mathbf{Y}^{(2)} & \mathbf{Y}^{(3)}
\end{array}\right]^{\mathrm{T}} \quad \mathbf{D}=\left[\begin{array}{lll}
\mathbf{D}^{(1)} & \mathbf{D}^{(2)} & \mathbf{D}^{(3)}
\end{array}\right]^{\mathrm{T}}
$$

In equation (3.5), $\alpha$ is the regularization parameter. The objective function $\Pi$ should be minimized to find the unknown vector $\mathbf{X}$. By taking the derivative of $\Pi$ with respect to $\mathbf{X}$ and equating to zero, the following equation is obtained

$$
\frac{\partial \Pi}{\partial \mathbf{X}}=-2 \mathbf{S}^{\mathrm{T}}(\mathbf{Y}-\mathbf{D})+2 \alpha \mathbf{X}=0
$$

In Eq. (3.7), $\mathbf{S}$ is the sensitivity matrix and is defined as follows

$$
\mathbf{S}^{(L)}=\left[\begin{array}{cccc}
S_{11}^{(L)} & S_{12}^{(L)} & \cdots & S_{1 q}^{(L)} \\
S_{21}^{(L)} & S_{22}^{(L)} & \cdots & S_{2 q}^{(L)} \\
\vdots & \vdots & \ddots & \vdots \\
S_{N_{L} 1}^{(L)} & S_{N_{L} 2}^{(L)} & \cdots & S_{N_{L} q}^{(L)}
\end{array}\right]
$$

where $L$ is the number of the load case and $q$ is the number of unknowns or the number of components of $\mathbf{X}$.

The components of the sensitivity matrix are defined as follows

$$
S_{i j}^{(L)}=\frac{\partial D_{i}^{(L)}}{\partial X_{j}}
$$

In order to compute the constants in an iterative process and calculate the sensitivity matrix, a program is written in python for the finite element software ABAQUS. This program uses the finite difference method to calculate the sensitivity matrix. For this purpose, each material constant is changed by a very small value and displacements at sampling points are obtained. The differences between these displacements are used to compute the sensitivity coefficients. The sensitivity coefficient $S_{i j}^{(L)}$ is computed through the following finite difference equation

$$
S_{i j}^{(L)}=\frac{D_{i}^{(L)}\left(X_{1}, \ldots, X_{j}+\mu X_{j}, \ldots, X_{q}\right)-D_{i}^{(L)}\left(X_{1}, \ldots, X_{q}\right)}{\mu X_{j}}
$$

where $\mu$ is a small value such as 0.001 . 
For example, if there are four unknown material constants, the sensitivity of the displacement at sampling point 3 with respect to material constant 2 is computed as follows

$$
S_{32}^{(2)}=\frac{D_{3}^{(2)}\left(X_{1}, X_{2}+\mu X_{2}, X_{3}, X_{4}\right)-D_{3}^{(2)}\left(X_{1}, X_{2}, X_{3}, X_{4}\right)}{\mu X_{2}}
$$

It is possible to obtain the unknown vector $\mathbf{X}$ through equation (3.7). Let the vector of unknown constants in the current step of the iterative process is represented by $\mathbf{X}_{c}$ and the corresponding displacement vectors for load cases 1,2 , and 3 are represented by $\mathbf{D}_{c}^{(1)}, \mathbf{D}_{c}^{(2)}$ and $\mathbf{D}_{c}^{(3)}$, respectively. The total displacement vector $\mathbf{D}_{c}$ is defined as follows

$$
\mathbf{D}_{c}=\left[\begin{array}{lll}
\mathbf{D}_{c}^{(1)} & \mathbf{D}_{c}^{(2)} & \mathbf{D}_{c}^{(3)}
\end{array}\right]^{\mathrm{T}}
$$

The total displacement vector in the new step, i.e. D, can be expressed as follows

$$
\mathbf{D}=\mathbf{D}_{c}+\mathbf{S}\left(\mathbf{X}-\mathbf{X}_{c}\right)
$$

where $\mathbf{X}$ is the vector of unknown constants in the new step. By using Eqs. (3.13) and (3.7) the following equation is obtained

$$
\mathbf{X}=\left[\mathbf{S}^{\mathrm{T}} \mathbf{S}+\alpha \mathbf{I}\right]^{-1}\left[\mathbf{S}^{\mathrm{T}}\left(\mathbf{Y}-\mathbf{D}_{c}\right)+\mathbf{S}^{\mathrm{T}} \mathbf{S} \mathbf{X}_{c}\right]
$$

Equation (3.14) is used iteratively through the following equation to obtain the constants

$$
\mathbf{X}^{k+1}=\left[\left(\mathbf{S}^{k}\right)^{\mathrm{T}} \mathbf{S}^{k}+\alpha^{k} \mathbf{I}\right]^{-1}\left[\left(\mathbf{S}^{k}\right)^{\mathrm{T}}\left(\mathbf{Y}-\mathbf{D}^{k}\right)+\left(\mathbf{S}^{k}\right)^{\mathrm{T}} \mathbf{S}^{k} \mathbf{X}^{k}\right]
$$

$k$ and $k+1$ are the numbers of iterations, and the convergence rule is defined as follows

$$
\left\|\mathbf{X}^{k+1}-\mathbf{X}^{k}\right\| \leqslant e
$$

where $e$ is the specified tolerance. In the cases where a large number of sampling points and/or many load cases are considered, selecting a small value or even zero for $\alpha$ results in stable solutions.

Inequality constraints on material constants (for example $0 \leqslant \kappa \leqslant 1 / 3$ ) are not considered in the inverse formulation of this study. In other words, the inverse formulation of this research uses an unconstrained optimization formulation. However, the obtained results must satisfy all the constraints. Otherwise, the problem has to be solved with different initial guesses.

\subsection{Initial guesses}

In order to start the iterative process and obtain the material constants, proper initial guesses are required. Selecting appropriate initial guesses reduces the number of iterations and increases the convergence rate. To find proper initial guesses for the unknown parameters of the hyper-elastic material, we try to find unknown Young's modulus $E$ and Poisson's ratio $\nu$ for an isotropic linear elastic material, which can approximately reconstruct the measured data for the original hyper-elastic material. For this purpose, the measured data $Y$ are used in a simple inverse analysis to find appropriate values for the two parameters of the pseudo linear elastic material. Then, the calculated parameters are used to compute initial guesses for the original material parameters. A similar approach has been used by Hematiyan et al. (2012) for providing initial guesses for identification of material constants of linear elastic anisotropic materials.

The pseudo material is assumed incompressible with $\nu=0.5$. The displacement vector obtained from the experiments on the hyper-elastic material, i.e. $\mathbf{Y}$, is assumed to be the displacement vector for the pseudo material with unknown $E$. The unknown parameter $E$ can be simply found as follows. 
The displacement vector at the sampling points of the pseudo material can be computed as

$$
\mathbf{D}=\frac{\overline{\mathbf{D}}}{E}
$$

where $\overline{\mathbf{D}}$ is the displacement vector at the same sampling points for a linear elastic material with $E=1 \mathrm{~Pa}$ and $\nu=0.5$. The vector $\overline{\mathbf{D}}$ can be computed using a direct analysis by the FEM. To find a suitable value of $E$, we minimize the difference between $\mathbf{D}$ and $\mathbf{Y}$. This can be carried out by minimizing the following expression

$$
F=\left(\mathbf{Y}-\frac{\overline{\mathbf{D}}}{E}\right)^{\mathrm{T}}\left(\mathbf{Y}-\frac{\overline{\mathbf{D}}}{E}\right)
$$

which gives

$$
E=\frac{\overline{\mathbf{D}}^{\mathrm{T}} \overline{\mathbf{D}}}{\overline{\mathbf{D}}^{\mathrm{T}} \mathbf{Y}}
$$

Now, we have found elastic constants of a pseudo linear elastic isotropic material for the inverse problem. After obtaining the Young modulus from equation (3.19), by selecting different values for $\varepsilon$, a set of stress-strain data is provided using the relation $\sigma=E \varepsilon$. This set of data is used to find initial guesses for the unknown constants of the original hyper-elastic material.

Consider a case of uniaxial loading with the axial stress $\sigma$ which causes the axial strain $\varepsilon$ in direction 1 for the pseudo material. The deformation of the material can be expressed as follows

$$
x_{1}=\lambda_{1} X_{1} \quad x_{2}=\lambda_{2} X_{2} \quad x_{3}=\lambda_{3} X_{3}
$$

where $\left(X_{1}, X_{2}, X_{3}\right)$ are rectangular Cartesian coordinates that identify material particles in some unstressed reference configuration, $\left(x_{1}, x_{2}, x_{3}\right)$ are the corresponding coordinates after deformation with respect to the same axes, and the coefficients $\left(\lambda_{1}, \lambda_{2}, \lambda_{3}\right)$ are principal stretches of the deformation. Therefore, the deformation gradient tensor $F_{i j}=\partial x_{i} / \partial X_{j}$ is expressed as follows

$$
\mathbf{F}=\left[\begin{array}{ccc}
\lambda_{1} & 0 & 0 \\
0 & \lambda_{2} & 0 \\
0 & 0 & \lambda_{3}
\end{array}\right]
$$

Substituting $B_{i j}=F_{i k} F_{j k}$ in equation (2.12), the following equation is obtained

$$
\sigma=2\left(\lambda_{1}^{2}-\frac{1}{\lambda_{1}}\right)\left(A_{10}+\frac{1}{\lambda_{1}} A_{01}\right)
$$

On the other hand, for the pseudo material we have

$$
\varepsilon=\frac{\partial\left(x_{1}-X_{1}\right)}{\partial X_{1}}=1-\lambda_{1}
$$

Substituting $\lambda_{1}=1+\varepsilon$ into Eq. (3.22) results in

$$
\sigma=2\left[(1+\varepsilon)^{2}-\frac{1}{1+\varepsilon}\right]\left(A_{10}+\frac{1}{1+\varepsilon} A_{01}\right)
$$

Equation (3.24) can be written in the following form

$$
Y=A_{10} X+A_{01}
$$


where $Y$ and $X$ are

$$
Y=\frac{\sigma(1+\varepsilon)}{2\left[(1+\varepsilon)^{2}-\frac{1}{1+\varepsilon}\right]} \quad X=1+\varepsilon
$$

By using the previously generated set of stress-strain data, a set of $X-Y$ data is computed using Eq. (3.26). Then a line is fitted through the $X-Y$ data by a simple linear regression. Considering Eq. (3.25) and by using the coefficients of the fitted line, the values of $A_{10}$ and $A_{01}$ are obtained. These values are considered as initial guesses for the inverse analysis.

A method to obtain the initial guesses for constants of anisotropic hyper-elastic materials is also presented here. These constants are $R_{10}, k_{1}, k_{2}$, and $\kappa$, see Eqs (2.17) and (2.18). Since $0 \leqslant \kappa \leqslant 1 / 3$, we simply select the value of 0.25 as an initial guess for $\kappa$. To simplify the process of obtaining the initial guesses we also set $k_{1}=k_{2}$. Therefore, only the initial guesses for $R_{10}$ and $k_{1}$ should be determined. Substituting Eqs. (2.17) and (2.18) into Eq. (2.16), the Cauchy stress is expressed as follows

$$
\boldsymbol{\sigma}=2 R_{10} \mathbf{B}+k_{1}\left(\frac{I_{1}}{4}+\frac{I_{4}}{4}-1\right) \exp \left[k_{1}\left(\frac{I_{1}}{4}+\frac{I_{4}}{4}-1\right)^{2}\right](\mathbf{B}+\mathbf{m} \otimes \mathbf{m})
$$

As discussed for the isotropic case, by using Eq. (3.19) Young modulus is obtained for the pseudo incompressible linear elastic material. Using the calculated Young's modulus and Hooke's law, the components of the stress tensor $\sigma$ and the strain tensor $\varepsilon$ at two different points are computed. The strain tensor is used in Eq. (2.2) to obtain the right Cauchy-Green deformation tensor $\mathbf{C}$ from which the deformation gradient tensor $\mathbf{F}$ and the tensor $\mathbf{B}$ can be obtained. Having $\mathbf{F}$ and $\mathbf{M}$, the vector $\mathbf{m}=\mathbf{F M}$ is also known and the unknowns in Eq. (3.27), i.e. $R_{10}$ and $k_{1}$, can be found. These computed values are considered as initial guesses for the corresponding parameters.

\section{Numerical examples}

In this Section, three examples are presented to show the efficiency of the presented method. In the two first examples, numerically simulated measured data are used; however, in the third example, the experimental data obtained from a polyvinyl alcohol sample are used for the identification of material parameters.

\subsection{Example 1. Isotropic hyper-elastic material}

In this example, a rectangular plate with a non-circular hole made of an isotropic hyper-elastic material is considered. As shown in Fig. 1, the body is subjected to three load cases. The loading location and directions vary in each case to obtain more useful measurement data. The measurement data were numerically simulated. For this purpose, three direct problems corresponding to the three load cases were solved by the exact material constants and displacement at sampling points where obtained. Then, some errors with a normal distribution were added to the displacement data to account for practical inaccuracies.

The exact material constants of the Mooney-Rivlin hyper-elastic model for the material are assumed $80 \mathrm{~Pa}$ and $20 \mathrm{~Pa}$ for $A_{10}$ and $A_{01}$, respectively. The initial guesses for the material constants obtained from the method presented in Section 3.2, are $A_{10}=90.34 \mathrm{~Pa}$ and $A_{01}=3.497 \mathrm{~Pa}$. These initial guesses are used in the developed program, and by using equation (3.15) the unknowns are updated in each step until they satisfy convergence rule.

At first, the problem was solved without any measurement error. Table 1 shows the number of iterations and obtained values for the constants when the results of the load cases where used separately $(1,2$ or 3$)$ or together $(1+2$ or $1+2+3)$ for the inverse analysis. The tolerance 


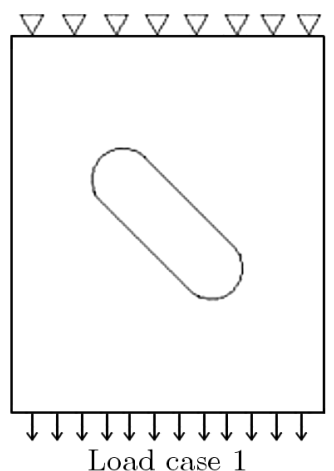

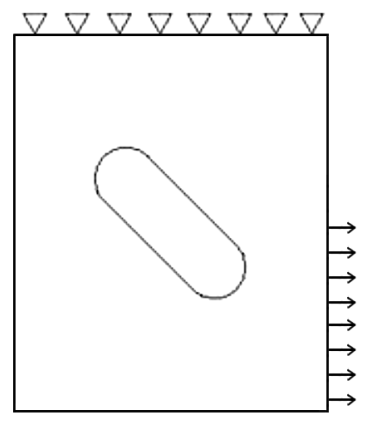

Load case 2

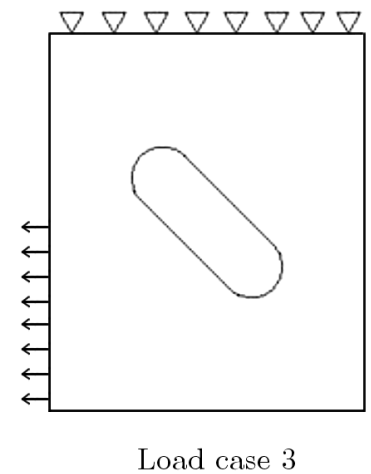

Load case 3

Fig. 1. Load cases applied to the material

for the convergence rule was assumed 0.01. Then the problem was solved by applying 1 and 5 percent error in the measurement data. These errors are applied in the exact solution of the problem using the normal distribution function. The results of these cases are shown in Table 1 as well. The convergence process for the case with $5 \%$ measurement error is shown in Fig. 2 . From the results of Table 1, it is clear that when there is no error in the measurements, the solution for all load cases converge easily to the material constants and the number of iterations is almost the same whether the load cases are solved together or separately. Further, when the error percentage in measurements increases, the number of iterations and errors in the obtained results increase too. In addition, the number of iterations is less for the cases that the problem is solved by using 2 or 3 load cases. Moreover, it is seen that the obtained results are more accurate when more than one load case is considered in the inverse analysis.

Table 1. Results for material constants with different values of measurement errors, Example 1

\begin{tabular}{|c|c|c|c|c|c|c|}
\hline \multicolumn{2}{|r|}{ Load case } & 1 & 2 & 3 & $1+2$ & $1+2+3$ \\
\hline \multirow{3}{*}{$\begin{array}{l}\text { without } \\
\text { measu- } \\
\text { rement } \\
\text { error }\end{array}$} & No. of iterations & 6 & 6 & 6 & 5 & 5 \\
\hline & Constants $[\mathrm{Pa}]$ & $\begin{array}{c}A_{10}=80 \\
(0)\end{array}$ & $\begin{array}{c}A_{10}=80 \\
(0)\end{array}$ & $\begin{array}{c}A_{10}=80 \\
(0)\end{array}$ & $\begin{array}{c}A_{10}=80 \\
(0)\end{array}$ & $\begin{array}{c}A_{10}=80 \\
(0)\end{array}$ \\
\hline & (Error [\%]) & $\begin{array}{c}A_{01}=20 \\
(0)\end{array}$ & $\begin{array}{c}A_{01}=20 \\
(0)\end{array}$ & $\begin{array}{c}A_{01}=20 \\
(0)\end{array}$ & $\begin{array}{c}A_{01}=20 \\
(0)\end{array}$ & $\begin{array}{c}A_{01}=20 \\
(0)\end{array}$ \\
\hline \multirow{3}{*}{$\begin{array}{c}1 \% \\
\text { measu- } \\
\text { rement } \\
\text { error }\end{array}$} & No. of iterations & 8 & 12 & 8 & 6 & 7 \\
\hline & Constants $[\mathrm{Pa}]$ & $\begin{array}{c}A_{10}=77.17 \\
(3.5)\end{array}$ & $\begin{array}{c}A_{10}=80.36 \\
(0.4)\end{array}$ & $\begin{array}{c}A_{10}=77.66 \\
(2.9)\end{array}$ & $\begin{array}{c}A_{10}=81.15 \\
(1.4)\end{array}$ & $\begin{array}{c}A_{10}=80.81 \\
\text { (1) }\end{array}$ \\
\hline & (Error [\%]) & $\begin{array}{c}A_{01}=23.18 \\
(15.9)\end{array}$ & $\begin{array}{c}A_{01}=19.52 \\
(2.4)\end{array}$ & $\begin{array}{c}A_{01}=22.15 \\
(10.7)\end{array}$ & $\begin{array}{c}A_{01}=18.74 \\
\quad(6.3)\end{array}$ & $\begin{array}{c}A_{01}=19.04 \\
\quad(4.8)\end{array}$ \\
\hline \multirow{3}{*}{$\begin{array}{l}5 \% \\
\text { measu- } \\
\text { rement } \\
\text { error }\end{array}$} & No. of iterations & \begin{tabular}{|l|}
10 \\
\end{tabular} & 11 & 17 & 4 & 5 \\
\hline & Constants $[\mathrm{Pa}]$ & $\begin{array}{c}A_{10}=70.058 \\
(12.4)\end{array}$ & $\begin{array}{c}A_{10}=72.97 \\
(8.7)\end{array}$ & $\begin{array}{c}A_{10}=75.66 \\
(5.4)\end{array}$ & $\begin{array}{c}A_{10}=86.43 \\
(8)\end{array}$ & $\begin{array}{c}A_{10}=80.53 \\
(0.6)\end{array}$ \\
\hline & (Error [\%]) & $\begin{array}{c}A_{01}=31.09 \\
\quad(55.4)\end{array}$ & $\begin{array}{c}A_{01}=25.93 \\
(29.6)\end{array}$ & $\begin{array}{c}A_{01}=25.18 \\
(25.9)\end{array}$ & $\begin{array}{c}A_{01}=12.60 \\
(36.9)\end{array}$ & $\begin{array}{c}A_{01}=19.39 \\
(3)\end{array}$ \\
\hline
\end{tabular}

\subsection{Example 2. Anisotropic hyper-elastic material}

In this example, the same geometry of the previous example but with an anisotropic hyper-elastic material is considered. The exact material constants of the Holzapfel hyper-elastic model for this material are assumed 7.64 Pa, 996.6, 524.6 and 0.226 for $R_{10}, k_{1}, k_{2}$ and $\kappa$, respectively. The direction of the fibers in a hyper-elastic material that determines the $\mathbf{M}$ and $\mathbf{M}^{\prime}$ vectors can be obtained from two-dimensional images of soft biological tissues (Schriefl et al., 2012). In this 
(a)

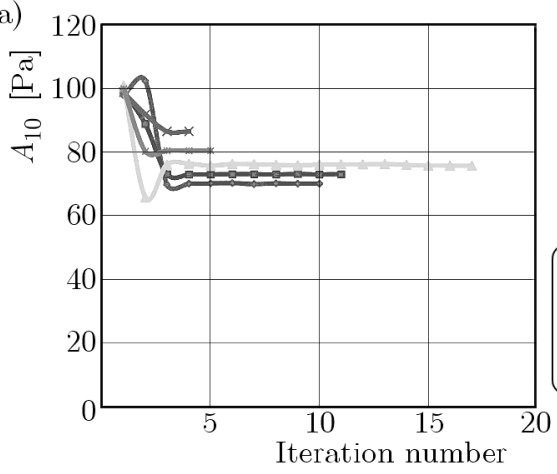

(b)

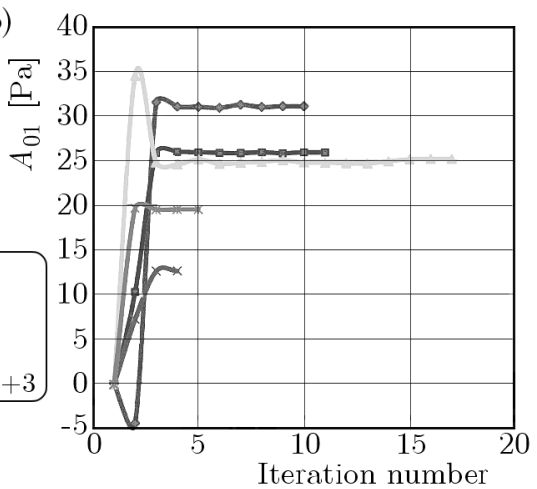

Fig. 2. Convergence of (a) $A_{10}$ and (b) $A_{01}$ for different load cases with $5 \%$ error in measurements

example, these vectors are assumed to be known $\left(\mathbf{M}=0.766 \mathbf{e}_{1}-0.643 \mathbf{e}_{2}, \mathbf{M}^{\prime}=0.5 \mathbf{e}_{1}-0.866 \mathbf{e}_{2}\right)$. The initial guesses for the material constant, which are obtained from the method presented in Section 3.2 , are $R_{10}=10.65 \mathrm{~Pa}, k_{1}=k_{2}=144.2$ and $\kappa=0.25$. At first, the problem was solved without any measurement error. Table 2 shows the number of iterations and obtained constants when the results of different load cases were used separately or together. The tolerance for the convergence rule was assumed 0.01. Then, the problem was solved by considering 1 and 5 percent error in the measurement data. The results of these cases are shown in Table 2 . The convergence process of constants for the case with $1 \%$ error in measurements is shown in Fig. 3. From the results given in Table 2, it is seen that the inverse solution diverges for load cases 2 and 3 even without any measurement error. Further, it is seen that when an error exists in measurements, the solution cannot be obtained by only one load case. It is also observed that the number of iterations for the solution with 3 load cases is smaller than the solution with 2 load cases.

Table 2. Results for material constants with different values of measurement errors, Example 2

\begin{tabular}{|c|c|c|c|c|c|c|}
\hline \multicolumn{2}{|c|}{ Load case } & 1 & 2 & 3 & $1+2$ & $1+2+3$ \\
\hline \multirow[b]{2}{*}{$\begin{array}{l}\text { without } \\
\text { measu- } \\
\text { rement } \\
\text { error }\end{array}$} & No. of iterat. & 7 & & & 7 & 8 \\
\hline & $\begin{array}{l}\text { Constants } \\
\text { (Error [\%]) }\end{array}$ & $\begin{array}{c}R_{10}=7.640 \mathrm{~Pa}(0) \\
k_{1}=996.6(0) \\
k_{2}=524.6(0) \\
\kappa=0.226(0)\end{array}$ & $\begin{array}{l}0 \\
0 \\
0 \\
0 \\
. \\
. \\
0\end{array}$ & 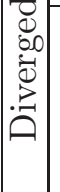 & $\begin{array}{c}R_{10}=7.640 \mathrm{~Pa}(0) \\
k_{1}=996.6(0) \\
k_{2}=524.6(0) \\
\kappa=0.226(0)\end{array}$ & $\begin{array}{c}R_{10}=7.640 \mathrm{~Pa}(0) \\
k_{1}=996.6(0) \\
k_{2}=524.6(0) \\
\kappa=0.226(0)\end{array}$ \\
\hline \multirow[b]{2}{*}{$\begin{array}{c}1 \% \\
\text { measu- } \\
\text { rement } \\
\text { error }\end{array}$} & No. of iterat. & & & & 18 & 7 \\
\hline & $\begin{array}{l}\text { Constants } \\
\text { (Error [\%]) }\end{array}$ & Diverged & $\ddot{\theta}$ & 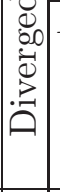 & $\begin{array}{c}R_{10}=7.645 \mathrm{~Pa}(0.06) \\
k_{1}=1015(1.8) \\
k_{2}=528.8(0.8) \\
\kappa=0.227(0.4)\end{array}$ & $\begin{array}{c}R_{10}=7.645 \mathrm{~Pa}(0.06) \\
k_{1}=1020(2.3) \\
k_{2}=527.3(0.5) \\
\kappa=0.227(0.4)\end{array}$ \\
\hline \multirow[b]{2}{*}{$\begin{array}{c}5 \% \\
\text { measu- } \\
\text { rement } \\
\text { error }\end{array}$} & No. of iterat. & & & & 37 & 15 \\
\hline & $\begin{array}{l}\text { Constants } \\
\text { (Error [\%]) }\end{array}$ & Diverged & & $\mid \begin{array}{c}0 \\
0 \\
0 \\
0 \\
. \\
. \\
0 \\
0\end{array}$ & $\begin{array}{c}R_{10}=7.607 \mathrm{~Pa}(0.4) \\
k_{1}=1079(8.2) \\
k_{2}=509.8(2.8) \\
\kappa=0.228(0.8)\end{array}$ & $\begin{array}{c}R_{10}=7.650 \mathrm{~Pa}(0.1) \\
k_{1}=926.0(7) \\
k_{2}=572.8(9.1) \\
\kappa=0.225(0.4)\end{array}$ \\
\hline
\end{tabular}

\subsection{Example 3. A rectangular plate with a hole made of polyvinyl alcohol}

In order to verify the proposed method for obtaining material constants of hyper-elastic materials, a sample was made of polyvinyl alcohol which exhibits hyperelastic mechanical behavior. Polyvinyl alcohol is usually used to simulate soft tissues of human body (Hebden et al., 
(a)

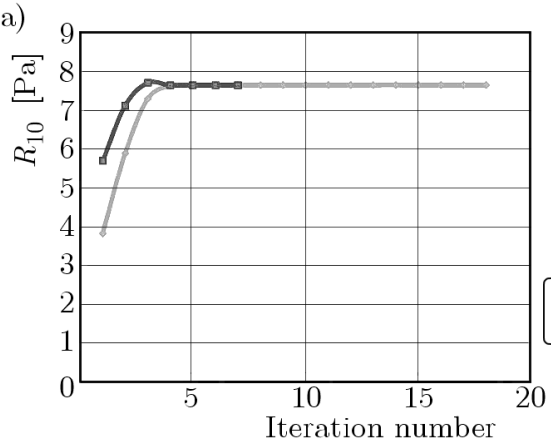

(c)

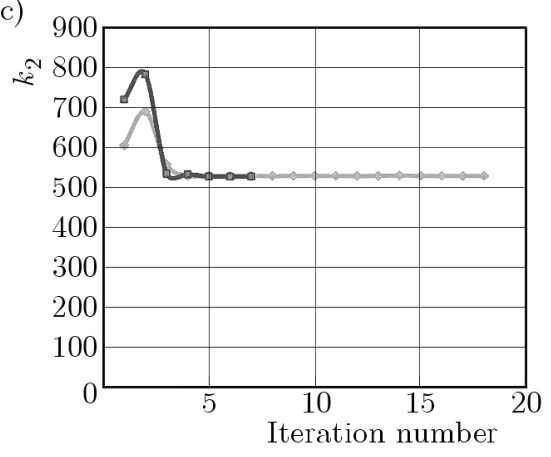

(b)

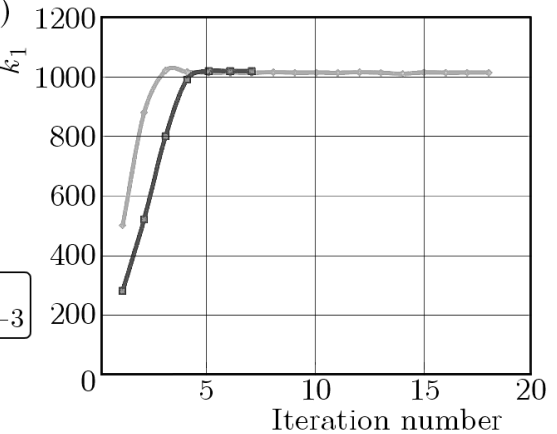

(d)

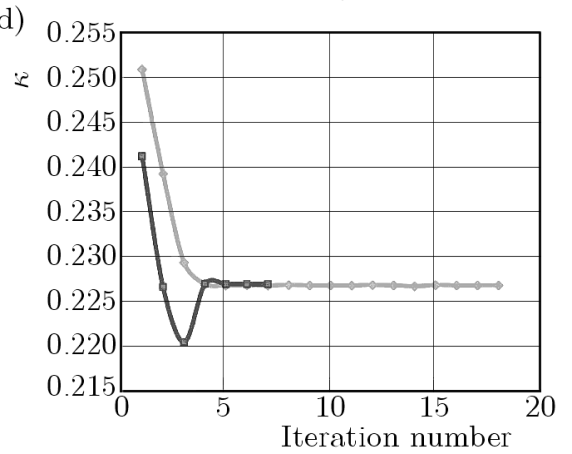

Fig. 3. Convergence of (a) $R_{10}$, (b) $k_{1}$, (c) $k_{2}$ and (d) $\kappa$ for different load cases with $1 \%$ error in measurements

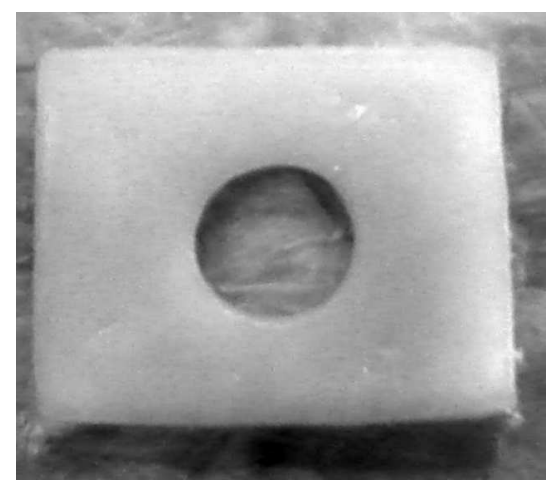

Fig. 4. The polyvinyl alcohol sample used in the experiments

2006). The sample used in the experiments (Fig. 4) is a rectangular plate with dimensions of $80.04 \mathrm{~mm} \times 10.02$ containing a $33.28 \mathrm{~mm}$ diameter hole.

Two load cases are considered for the polyvinyl alcohol sample. In the first case, the sample is placed horizontally on the desk and by using a force meter, a load is applied to one end while the other end is fixed. This load case is shown in Fig. 5. In this case, a load of $1.2 \mathrm{~N}$ is applied to the sample.

In the second case, the sample is hanged by its weight and by using a hook, a load is applied to the sample. This case is shown in Fig. 6. A load of $1.5 \mathrm{~N}$ is applied in this case. It should be mentioned that, in this case, there exists a body force in the same direction of loading too. Therefore, the deflection of the member in the second case is much more than the deflection in the first load case.

For measuring the displacement at the sampling points, these points were marked before the test. By using the measured displacements, the material constants are obtained for a simple cylindrical sample and for the rectangular sample with a hole. The finite element model (con- 


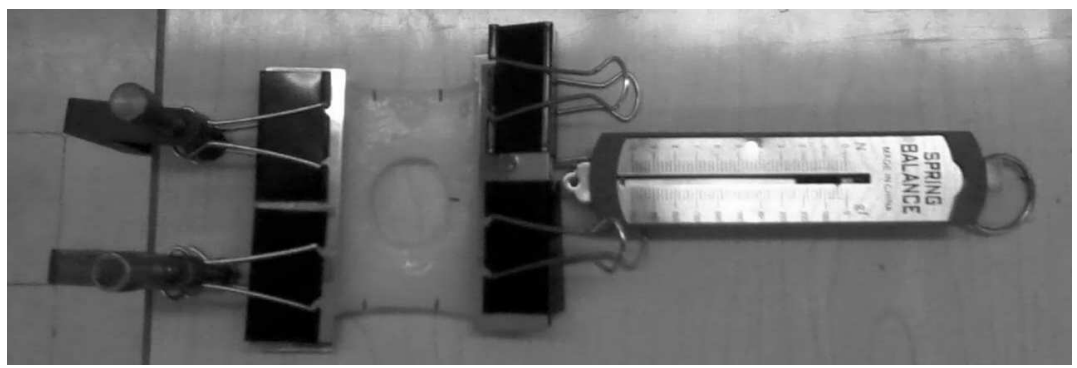

Fig. 5. First load case for the rectangular sample

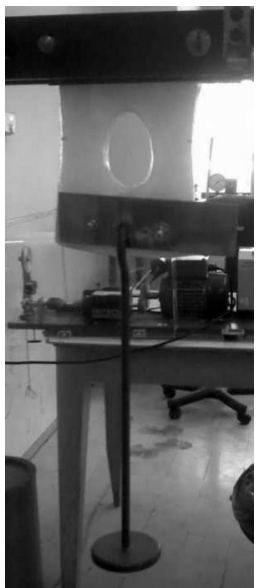

Fig. 6. Second load case for the rectangular sample

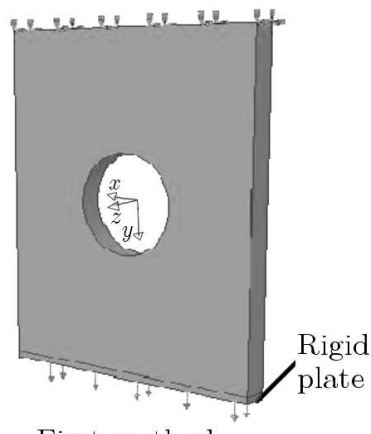

First method

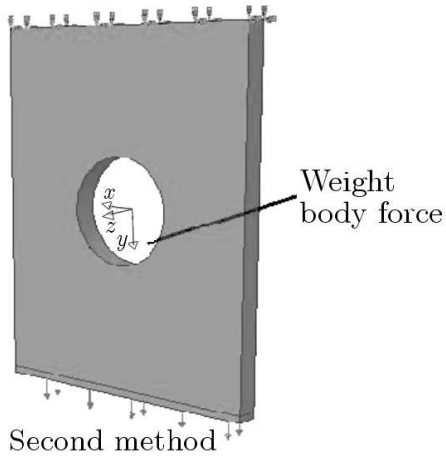

Second method

Fig. 7. Load application in FEA for the two cases

structed in ABAQUS) and displacement for the two load cases are shown in Figs. 7 and 8. As it is shown in Fig. 7, the load is not directly applied to the material. A rigid plate is used in the FEA model for load application in order to simulate the experiment more accurately.

The material constants obtained from loading the cylindrical sample are presented in Table 3. The material constants obtained for the rectangular sample are presented in Table 4, where the results of the first and second cases are used separately and together. From Table 4 it is seen that when each test is used separately to obtain the material constants, the number of iterations is larger and the constants deviate more from those obtained for the cylindrical sample in Table 3. Further, when two tests are used together to obtain the constants, the deviation from constants of Table 3 and number of iterations are smaller. 


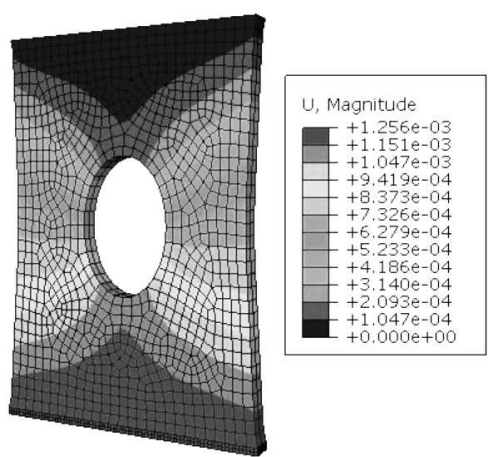

First method

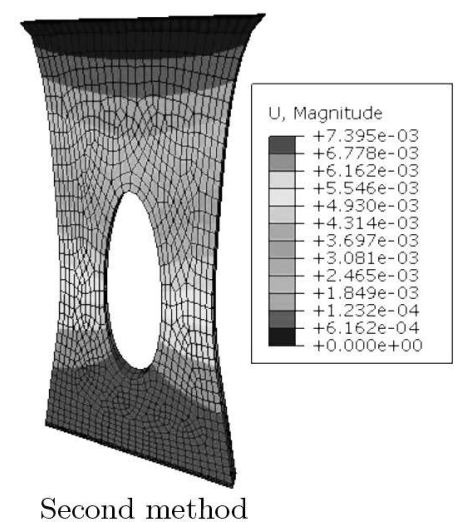

Second method

Fig. 8. Displacement contours in the sample for two load cases

Table 3. Material constants obtained for the cylindrical sample

\begin{tabular}{|c|c|c|}
\hline$C_{10}[\mathrm{~Pa}]$ & $C_{01}[\mathrm{~Pa}]$ & Number of iterations \\
\hline \hline 4.964 & 0.541 & 19 \\
\hline
\end{tabular}

Table 4. Material constants obtained for the rectangular sample with a hole

\begin{tabular}{|c|c|c|c|}
\hline Test & $C_{10}[\mathrm{~Pa}]$ & $C_{01}[\mathrm{~Pa}]$ & Number of iterations \\
\hline \hline 1 & 6.231 & 0.756 & 28 \\
\hline 2 & 5.843 & 0.641 & 43 \\
\hline $1+2$ & 5.006 & 0.524 & 13 \\
\hline
\end{tabular}

\section{Conclusions}

A method to obtain the constants of isotropic and anisotropic incompressible hyper-elastic materials is presented. In the proposed method, by using the measured displacements at some sampling points on the boundary of a member with a non-standard shape, the unknown material constants of the material are computed. The measured data are obtained from more than one test with different load cases. It is shown that the method is more efficient when the results of two or three load cases are simultaneously used to solve the problem.

From the results of the numerical examples, it is concluded that when the measurement error is zero for isotropic materials, the solution process corresponding to all load cases easily converge to the exact solution and the number of iterations is almost the same whether the load cases are solved together or separately. However, for anisotropic hyper-elastic materials, even in the cases without any measurement error, the solution corresponding to some load cases may diverge. Since the number of constants to be determined in the anisotropic case is more than the isotropic case, more load cases are needed to find the unknown constants of hyper-elastic anisotropic materials.

For both isotropic and anisotropic hyper-elastic materials, when the measurement error increases, the number of iterations and the error in the obtained results increase too. Further, it can be seen that usually the number of iterations is smaller for the cases that the problem is solved by using the measured data from 2 or 3 load cases and the results obtained are more accurate.

In this research, the preferred direction vectors are considered to be known. However, if one considers these parameters as unknowns, the number of the unknowns of the inverse problem increases. This will complicate the problem more and more, and the need for conducting more experiments increases as well. 


\section{References}

1. Abyaneh M.H., Wildman R.D., Ashcroft I.A., Ruiz P.D., 2013, A hybrid approach to determining cornea mechanical properties in-vivo using a combination of nano-indentation and inverse finite element analysis, Journal of the Mechanical Behavior of Biomedical Materials, 27, $239-248$

2. Aнn B., Kiм Y., Kiм J., 2008, Biomechanical characterization with inverse FE model parameter estimation: Macro and Micro applications, International Conference on Control, Automation and Systems, 1769-1772

3. BAKer M., Shrot A., 2013, Inverse parameter identification with finite element simulation using knowledge-based descriptors, Computational Materials Science, 69, 128-136

4. Balaraman K., Mukherjee S., Chawla A., Malhotra R., 2005, Inverse finite element characterization of soft tissue using impact experiments and Taguchi method, SAE Internationals, 2005-01-4044

5. Czabanowski R., 2010, Experimental identification of hyperelastic material parameters for calculations by the finite element method, Journal of KONES, 17, 1, 87-92

6. Fung Y.C., 1993, Biomechanics: Mechanical Properties of Living Tissues, Springer, New York

7. Gasser T.C., Ogden R.W., Holzapfel G.A., 2006, Hyperelastic modeling of arterial layers with distributed collagen fiber orientations, Journal of Royal Society Interface, 3, 6, 15-35

8. Hartman S., 2001, Numerical studies on the identification of the material parameters of Rivlin's hyper-elasticity using tension-torsion tests, Acta Mechanica, 148, 129-155

9. Hebden J.C., Price B.D., Gibson A.P., Royle G., 2006, A soft deformable tissue equivalent phantom for diffuse optical tomography, Physics in Medicine and Biology, 51, 21, 5581-5590

10. Hematiyan M.R., Khosravifard A., Siah Y.C., Tan C.L., 2012, Identification of material parameters of two-dimensional anisotropic bodies using an inverse multi loading boundary element technique, Computer Modeling in Engineering and Science, 87, 1, 55-76

11. Holzapfel G.A., Gasser T.C., 2000, A new constitutive framework for arterial wall mechanics and a comparative study of material models, Journal of Elasticity, 61, 1-48

12. Holzapfel G.A., 2000, Nonlinear Solid Mechanics, Technical University Graz, Austria

13. Holzapfel G.A., Ogden R.W., 2010, Constitutive modeling of arteries, Journal of Royal Society, 466, 2118, 1551-1597

14. Hu T., Desai J.P., 2004, Characterization of Soft-Tissue Material Properties: Large Deformation Analysis, Drexel University, Philadelphia

15. Krouskop T.A., Wheeler T.M., Kallel F., Garra B.S. Hall T., 1998, Elastic moduli of breast and prostate tissues under compression, Ultrasonic Imaging, 20, 260-274

16. Liu G.R., HAN X., 2003, Computational Inverse Techniques in Nondestructive Evaluation, CRC Press, Boca Raton, London, New York, Washington, D.C.

17. Miller K., Chinzei K., 1997, Constitutive modeling of brain tissue: experiment and theory, Journal of Biomechanics, 30, 1115-1121

18. Mehrabian H., 2008, Soft tissue hyper-elastic parameter reconstruction for breast cancer assessment, Mastered Engineering Science Thesis, University of Toronto

19. Mesa-Múnera E., Ramírez-Salazar J.F., Boulanger P., Branch J. W., 2012, Inverse-FEM characterization of a brain tissue phantom to simulate compression and indentation, Ingeniería y Cienc, 8, 11-36

20. Moulton M.J., Creswell L.L., Actis R.L., Myers K.W., Vannier M.W., Szabo B.A., PASQUE M.K., 1995, An inverse approach to determining myocardial material properties, Journal of Biomechanics, 28, 8, 935-948 
21. Ogden R.W., Saccomandi G., Sgura I., 2004, Fitting hyper-elastic models to experimental data, Computational Mechanics, 34, 6, 484-502

22. Pamidi M.R., Advani S.H., 1978, Nonlinear constitutive relations for human brain tissue, Journal of Biomechanical Engineering, 100, 1, 44-48

23. Rauchs G., Bardon J., Georges D., 2010, Identification of the material parameters of a viscous hyper-elastic constitutive law from spherical indentation tests of rubber and validation by tensile tests, Mechanic of Materials, 42, 961-973

24. Rivlin R.S., SAunder D.W., 1951, Large elastic deformations of isotropic materials VII. Experiments on the deformation of rubber, Journal of Royal Society, 243, 865, 251-288

25. Schriefl A.J., Reinisch A.J., Sankaran S., Pierce D.M., Holzapfel G.A., 2012, Quantitative assessment of collagen fibre orientations from two-dimensional images of soft biological tissues, Journal of Royal Society, 9, 76, 3081-3093

26. Unnikrishnan V.U., Unnikrishnan G.U., Reddy J.N., 2012, Biomechanics of breast tumor: effect of collagen and tissue density, International Journal of Mechanics and Materials in Design, $\mathbf{8}, 3,257-267$

Manuscript received August 25, 2014; accepted for print May 8, 2015 